\title{
Audiovestibular symptoms and sequelae in COVID-19 patients
}

\author{
Roberto Gallus $^{\mathrm{a}}$, Andrea Melis ${ }^{\mathrm{b}}$, Davide Rizzo ${ }^{\mathrm{b}, *}$, Antonio Piras ${ }^{\mathrm{b}}$, Laura Maria De Luca ${ }^{\mathrm{b}}$, \\ Pierangela Tramaloni ${ }^{\mathrm{b}}$, Antonello Serra ${ }^{\mathrm{c}}$, Eleonora Longoni ${ }^{\mathrm{d}}$, \\ Giovanni Maria Soro ${ }^{\mathrm{e}}$ and Francesco Bussu ${ }^{\mathrm{b}, \mathrm{f}}$ \\ ${ }^{a}$ Otolaryngology, Mater Olbia Hospital, Olbia, Italy \\ ${ }^{\mathrm{b}}$ Otolaryngology Division, Azienda Ospedaliero Universitaria, Sassari, Italy \\ ${ }^{\mathrm{c}}$ Surveillance and Prevention Department, University Hospital of Sassari, Sassari, Italy \\ ${ }^{\mathrm{d}}$ Unit of Otorhinolaryngology, Department of Surgery, Azienda Ospedaliero - Universitaria di Cagliari, \\ University of Cagliari, Cagliari, Italy \\ ${ }^{\mathrm{e}}$ Directorate General, Azienda Ospedaliero Universitaria, Sassari, Italy \\ ${ }^{\mathrm{f}}$ Department of Medical, Surgical and Experimental Science, University of Sassari, Sassari, Italy
}

Received 27 May 2020

Accepted 20 January 2021

\begin{abstract}
.
BACKGROUND: since the beginning of COVID-19 outbreak a growing number of symptoms and deficits associated with the new pathology have emerged, among them cochlear damage in otherwise asymptomatic COVID-19 patients has been described.

OBJECTIVE: to investigate general and audiovestibular symptoms and sequelae in healed patients, and to seek for any sign of residual or permanent hearing or vestibular loss.

METHODS: we reviewed the data coming from 48 Covid-19 patients whose nasopharyngeal swabs have turned negative, all employed at our facility, that opted in for a free screening of audiovestibular symptoms offered by our hospital after the aforementioned report was published. The screening included a tonal pure tone audiometry, a vHIT and SHIMP test, as well as a survey including known symptoms and audiovestibular symptoms.

RESULTS: general symptoms as reported by our patients largely reflect what reported by others in the literature. 4 (8.3\%) patients reported hearing loss, 2 (4.2\%) tinnitus, 4 dizziness $(8.3 \%), 1$ spinning vertigo (2\%), 1 dynamic imbalance (2\%), 3 static imbalance $(6.3 \%)$. Most audiovestibular symptoms have regressed. Thresholds at pure tone audiometry and vHIT gain were within normality range in all post-Covid-19 patients.

CONCLUSIONS: even if some patients suffer from audiovestibular symptoms, these are mostly transitory and there is no clear evidence of clinically relevant persistent cochlear or vestibular damage after recovery.
\end{abstract}

Keywords: vHIT, pure tone audiometry, covid-19, audiovestibular symptoms, general symptoms

\section{Introduction}

Since the beginning of the COVID-19 outbreak [3, $4,8,21]$, and subsequent declaration of pandemic status by the WHO [22], thousand of research groups

\footnotetext{
*Corresponding authors. Davide Rizzo, MD, PhD, Otolaryngology Division, Azienda Ospedaliera Universitaria di Sassari, Viale an Pietro 07100 Sassari, Italy. Tel.: +393492931723; E-mail: davide.rizzo@aousassari.it.
}

all over the world have profused an enormous and probably unprecedented collective effort in order to retrieve as much data and scientific knowledge as possible both on COVID-19 and the responsible virus: Sars-Cov-2 [23]. Among the relevant results, an ever growing list of presenting and associated symptoms has been described, allowing for an early diagnosis in atypical cases, self awareness of potentially infected people, and better care of symptoms and lesions that would otherwise pass unnoticed [15]. 
Along with the early described and commonest respiratory and flu-like symptoms (fever $88.5 \%$, cough $68.6 \%$, myalgia/fatigue $35.8 \%$, expectoration $28.2 \%$, dyspnea $21.9 \%$, sore throat up to $44.4 \%$ and nasal congestion up to $25 \%$ ) $[7,10]$, some interesting clinical presentations often seen by specialists and not general practitioners have emerged. The list includes GI symptoms (diarrhea, nausea, vomiting, abdominal pain) in $8,7 \%$ of patients according to a recent meta-analysis (range 3-39.6\% [7, 14, 19, 20]; ophthalmologic (conjuntivitis) in $1.1 \%$ of patients [9]; neurological symptoms in $36.4 \%$ of patients, including central nervous system manifestations (dizziness $16.8 \%$, headache $13.1 \%$, impaired consciousness $7.5 \%$, acute cerebrovascular disease $2.8 \%$, ataxia $0.5 \%$, and seizure $0.5 \%$ ), peripheral nervous system manifestations (taste impairment $5.6 \%$, smell impairment $5.1 \%$, vision impairment $1.4 \%$, and nerve pain 2.3 ), and skeletal muscular injury manifestations $10.7 \%$ [11]. Notably dizziness, taste impairment and smell impairment are symptoms usually first seen and evaluated by otolaryngologists, and higher percentages of these symptoms can be detected through specific tests usually performed by otolaryngologists $[1,5,6,18]$.

Another potentially neglected symptom of COV ID-19, namely hearing loss, has been recently reported [13]. Specifically the Author noted a difference on high frequencies pure tone threshold and TEO AEs amplitude between asymptomatic COVID-19 patients (tested during the active phase of the infection) and controls. He hypothesised that Sars-Cov2 may have deleterious effects on cochlear hair cell functions, despite the patient being asymptomatic.

Considering the evidence of potentially unnoticed hearing loss, and of other neurological symptoms described in the literature, we decided to offer to our hospital's personnel who previously contracted COVID-19 a free screening for audiovestibular symptoms and deficits. We also asked them to fill in an anamnestic questionnaire to highlight other potential neurological and general symptoms and sequelae. The present study is an analysis of the data collected during the screening.

\section{Material and methods}

Aim of the study was to assess the presence of persistent audiovestibular damage in healed COVID-19 patients, and the distribution and onset of other general and neurological symptoms. We retrospectively analysed the audiovestibular data of 48 COVID-19 patients, tested within 2 weeks from the second negative swab, and compared them with 28 age-sex matched voluntary controls from the same health workers population. All the patients were employees of our hospital that contracted COVID-19 and opted in for a free screening of audiovestibular symptoms, offered during the COVID-19 outbreak that heavily hit our Institution (Azienda Ospedaliera Universitaria) and our province (Sassari, Italy). The screening was performed between April and May. Inclusion criteria for the screening were: being employee of our hospital, and previous history of COVID-19, with 2 consecutive negative RT-PCR on nasopharyngeal swabs [16] (performed with an interval of at least 48 hours), in order to avoid unnecessary risk for the operators involved. Inclusion criteria for the subsequent retrospective study was acceptance of the informed consent, involving also treatment of personal data and anonymization. As for the control group, inclusion criteria were no previous history of audiovestibular symptoms and acceptance of the informed consent as well.

Therefore, before the instrumental tests anamnestic data were collected from all patients. A wide range of general, neurological and audiovestibular symptoms and their characteristics were systematically investigated (Table 1). When one or more symptoms were reported, the patient was asked to clarify if the symptom was still ongoing, if it started as a presenting symptom ("early onset"), or if it occurred during the disease or after the clinical and molecular remission ("late onset"). Before testing, all patients underwent an otological examination. Tympanometry and cochleo-stapedial reflexes testing (Clarinet, Inventis Biomedica) were performed to rule out middle ear pathology. Tonal audiometries (Amplaid A319 TDH) were performed by the same audiology technician, with a validated technique as described elsewhere [2]. Both air (from 250 to $8000 \mathrm{~Hz}$ ) and bone $(250$ to $4000 \mathrm{~Hz})$ conduction thresholds were measured. vHIT was performed always with the same instrument (EyeSeeCam, Interacoustics) by the same experienced otolaryngologist, following the manufacturer manuals and the on screen indications from the proprietary software (Interacoustics). Gain threshold for vestibular loss was set at any value lower than 0,6 at $60 \mathrm{~ms}$, as described elsewhere $[12,17]$.

\subsection{Statistical analysis}

Descriptive statistics, and ANOVA to compare audiological and vestibular data between patients and 
Table 1

Overview of reported symptoms. Total number of patients is 48 . Absolute number and percentage inside round brackets are reported. Early onset symptoms were those present at diagnosis, late onset those appeared during or toward the end of the clinical course of the pathology. Patients were specifically asked to report only symptoms of new onset or significant variations of pre-existing symptoms (applies only to audiovestibular symptoms). *stands for unknown data (patients didn't remember - were unsure)

\begin{tabular}{|c|c|c|c|c|c|c|}
\hline Symptom & Yes & No & Early onset & Late onset & Persistent & Regressed \\
\hline Fever & $16 / 48(33.3 \%)$ & $32 / 48(66.6 \%)$ & $*$ & $*$ & $0 / 16(0 \%)$ & $16 / 16(100 \%)$ \\
\hline Dyspnea & $9 / 48(18.7 \%)$ & $39 / 48(81.2 \%)$ & * & * & $0 / 9(0 \%)$ & $9 / 9(100 \%)$ \\
\hline Cough & $21 / 48(43.7 \%)$ & $27 / 48(56.2 \%)$ & $*$ & $*$ & $0 / 21(0 \%)$ & $21 / 21(100 \%)$ \\
\hline Thoracic pain & $8 / 48(16.6 \%)$ & $40 / 48(83.3 \%)$ & $*$ & $*$ & $0 / 8(0 \%)$ & $8 / 8(100 \%)$ \\
\hline Asthenia & $21 / 48(43.7 \%)$ & $27 / 48(56.2 \%)$ & $*$ & $*$ & $0 / 21(0 \%)$ & $21 / 21(100 \%)$ \\
\hline Myalgia & $17 / 48(35.4 \%)$ & $31 / 48(64.5 \%)$ & $*$ & $*$ & $0 / 17(0 \%)$ & $17 / 17(100 \%)$ \\
\hline Diarrhea & $15 / 48(31.2 \%)$ & $33 / 48(68.7 \%)$ & $*$ & $*$ & $0 / 15(0 \%)$ & $15 / 15(100 \%)$ \\
\hline Conjuntivitis & $4 / 48(8.3 \%)$ & $44 / 48(91.6 \%)$ & $*$ & $*$ & $0 / 4(0 \%)$ & $4 / 4(100 \%)$ \\
\hline General malaise & $19 / 48(39.5 \%)$ & $29 / 48(60.4 \%)$ & $*$ & $*$ & $0 / 19(0 \%)$ & $19 / 19(100 \%)$ \\
\hline Sore Throat & $17 / 48(35.4 \%)$ & $31 / 48(64.5 \%)$ & $*$ & $*$ & $0 / 17(0 \%)$ & $17 / 17(100 \%)$ \\
\hline Headache & $24 / 48(50 \%)$ & $24 / 48(50 \%)$ & $*$ & $*$ & $1 / 24(4.2 \%)$ & $23 / 24(95.8 \%)$ \\
\hline Cutaneous rash & $6 / 48(12.5 \%)$ & $42 / 48(87.5 \%)$ & $*$ & $*$ & $0 / 6(0 \%)$ & $6 / 6(100 \%)$ \\
\hline Hypo-Anosmia & $21 / 48(53.8 \%)$ & $27 / 48(56.2 \%)$ & $4 / 21(19.1 \%)$ & $17 / 21(80.9 \%)$ & $3 / 21(14.3 \%)$ & $18 / 21(85.7 \%)$ \\
\hline Hypo-Ageusia & $18 / 48(37.5 \%)$ & $30 / 48(62.5 \%)$ & $2 / 18(11.1 \%)$ & $16 / 18(88.9 \%)$ & $2 / 18(11.1 \%)$ & $16 / 18(88.9 \%)$ \\
\hline Tinnitus & $2 / 48(4.2 \%)$ & $46 / 48(95.8 \%)$ & $*$ & $*$ & $1 / 2(50 \%)$ & $1 / 2(50 \%)$ \\
\hline Hearing loss & $4 / 48(8.3 \%)$ & $44 / 48(91.6 \%)$ & $*$ & $*$ & $1 / 4(25 \%)$ & $3 / 4(75 \%)$ \\
\hline Dizziness & $4 / 48(8.3 \%)$ & $44 / 48(91.7 \%)$ & $0 / 4(0 \%)$ & $4 / 4(100 \%)$ & $0 / 4(0 \%)$ & $4 / 4(100 \%)$ \\
\hline Spinning vertigo & $1 / 48(2 \%)$ & $47 / 48(98 \%)$ & $0 / 1(0 \%)$ & $1 / 1(100 \%)$ & $0 / 1(0 \%)$ & $1 / 1(100 \%)$ \\
\hline $\begin{array}{l}\text { Static imbalance/ } \\
\text { disequilibrium }\end{array}$ & $3 / 48(6.3 \%)$ & $45 / 48(93.7 \%)$ & $2 / 3(66.7 \%)$ & $1 / 3(33.3 \%)$ & $1 / 3(33.3 \%)$ & $2 / 3(66.7 \%)$ \\
\hline $\begin{array}{l}\text { Dynamic imbalance/ } \\
\text { disequilibrium }\end{array}$ & $1 / 48(2 \%)$ & $47 / 48(98 \%)$ & $1 / 1(100 \%)$ & $0 / 1(0 \%)$ & $0 / 1(0 \%)$ & $1 / 1(100 \%)$ \\
\hline Head motion intolerance & $0 / 48(0 \%)$ & 48/48 (100\%) & * & $*$ & * & $*$ \\
\hline $\begin{array}{l}\text { Visually triggered } \\
\text { dizziness }\end{array}$ & $0 / 48(0 \%)$ & $48 / 48(100 \%)$ & $*$ & * & * & * \\
\hline
\end{tabular}


control group were performed. The comparison included single main frequencies from pure tone audiometry, cochleo-stapedial reflexes, PTA - average of $0.5,1,2$ and $4 \mathrm{kHz}$ for the two ears, and vHIT values. For all tests a statistically significant difference was assumed for $p<0.05$. Statistical analysis was performed using the JASP software, release 0.12 .2 .

\section{Results}

Mean age of the whole patient group was 45 (STD 9.6), 37 (77\%) were female, 11 (23\%) were male. Reported symptoms are summarized in Table 1 . None of the patients required hospitalization for Covid-19.

Controls were age and sex matched. 7 patients were excluded from the audiological comparison with the control group for a reportedly pre-existing hearing loss. Among the remaining 41 patients, 4 (8.3\%) reported subjective hearing loss and $2(4.2 \%)$ tinnitus. 1 patient with hearing loss and 1 with tinnitus reported persistent symptoms. Anyway, all the patients who reported transient or persistent tinnitus or hearing loss, had a normal hearing threshold at the time of our testing (ASHA classification [24]). 41 patients and 28 controls, were compared as for PTA and also by single frequency. No differences between patients and controls were recorded as for PTA $(p=0.094)$. The only frequency with significantly higher thresholds in former Covid-19 patients was $0.25 \mathrm{kHz}(p<0.016)$, while controls had significantly higher thresholds for $2 \mathrm{kHz}(\mathrm{p} \mathrm{0.042)}$ ) and $4 \mathrm{kHz}$ (p 0.029) (Table 2). The average difference between the two groups for the aforementioned frequencies was respectively $1.4 \mathrm{~dB}$, $1.6 \mathrm{~dB}$ and $3.2 \mathrm{~dB}$ which is definitely not clinically relevant.

3 patients were excluded from the vestibular comparison due to a pre-existing history of vertigo and vestibular damage. 45 patients and 28 controls, for a total of 146 vestibules were analysed. 5 patients $(10.4 \%)$ reported one or more vestibular symptoms, namely dizziness in 4 cases $(8.3 \%)$, spinning vertigo in 1 case $(2 \%)$, dynamic imbalance in one case $(2 \%)$ and static imbalance in 3 cases $(6.3 \%)$. Most of the symptoms had a late onset (at least 1 week after the diagnosis of Covid-19), except for dynamic and static imbalance mostly reported as early symptoms (Table 1). Most vestibular symptoms were transient and resolved at the time of the screening, only one case reported a persistent sensation of isolated static imbalance. Gain values for most patients with

Table 2

Statistical output of ANOVA test comparing Tonal Audiometry (dB), PTA (dB), Cochleo Stapedial reflexes $(\mathrm{dB})$, vHIT values for each canal and SHIMP between cases and controls. Significant values marked in bold

\begin{tabular}{lccc}
\hline Parameter & $\begin{array}{c}\text { Mean Value: } \\
\text { Cases - Controls }\end{array}$ & $\begin{array}{c}\text { Std. Deviation: } \\
\text { Cases - Controls }\end{array}$ & $p$ \\
\hline PTA & $10.503-11.585$ & $2.831-2.214$ & 0.094 \\
$0,25 \mathrm{kHz}$ & $11.585-10.179$ & $2.945-0.656$ & $\mathbf{0 . 0 1 6}$ \\
$0,5 \mathrm{kHz}$ & $12.073-10.893$ & $3.255-1.696$ & 0.083 \\
$1 \mathrm{kHz}$ & $9.695-10.268$ & $3.122-0.787$ & 0.346 \\
$2 \mathrm{kHz}$ & $9.573-11.250$ & $2.953-3.758$ & $\mathbf{0 . 0 4 2}$ \\
$4 \mathrm{kHz}$ & $10.671-13.929$ & $4.403-7.681$ & $\mathbf{0 . 0 2 9}$ \\
6 kHz & $11.707-14.464$ & $6.160-7.916$ & 0.109 \\
$8 \mathrm{kHz}$ & $12.012-16.339$ & $7.870-10.661$ & 0.057 \\
Ipsi 0,5 kHz & $89.610-89.732$ & $10.951-13.563$ & 0.967 \\
Ipsi 1 kHz & $88.659-90.625$ & $6.522-13.081$ & 0.412 \\
Ipsi 2 kHz & $90.610-91.875$ & $8.381-13.534$ & 0.633 \\
Ipsi 4 kHz & $95.549-95.714$ & $10.055-12.245$ & 0.951 \\
Contra 0,5 kHz & $92.927-93.750$ & $8.022-10.240$ & 0.710 \\
Contra 1 kHz & $92.439-94.554$ & $6.459-9.838$ & 0.284 \\
Contra 2 kHz & $92.439-92.232$ & $6.162-8.508$ & 0.907 \\
Contra 4 kHz & $100.366-100.714$ & $10.179-12.835$ & 0.901 \\
Gain 60 ms LSC Right & $0.856-0.836$ & $0.134-0.211$ & 0.629 \\
Gain 60 ms LSC Left & $0.883-0.838$ & $0.119-0.164$ & 0.197 \\
Gain 60 ms RA & $0.799-0.950$ & $0.256-0.120$ & $\mathbf{0 . 0 4 0}$ \\
Gain 60 ms LP & $0.857-0.854$ & $0.261-0.199$ & 0.967 \\
Gain 60 ms LA & $0.973-0.914$ & $0.167-0.177$ & 0.216 \\
Gain 60 ms RP & $0.920-0.993$ & $0.219-0.094$ & 0.139 \\
SHIMP Gain 60 ms LSC Right & $0.843-0.766$ & $0.167-0.270$ & 0.151 \\
SHIMP Gain 60 ms LSC Left & $0.818-0.745$ & $0.166-0.257$ & 0.159 \\
\hline
\end{tabular}


reported vestibular symptoms were normal, except for one patient that experienced transient dizziness, asymptomatic at the time of the screening, that had a gain reduction (0.45) of the left posterior semicircular canal at vHIT, but didn't show overt or covert saccades. None of the other subjects presented overt or covert saccades. When comparing gains between patients and controls the only significant difference has been recorded in the vHIT gain of the right anterior canal ( $\mathrm{p} 0.04)$ (Table 2, with an average difference of $0.15(+/-0.07)$.

\section{Discussion}

One of the most important aspects while investigating a novel disease as COVID-19, is to properly describe the associated symptoms. This allows for an early diagnosis, identification of cases with atypical presentation, and treatment of deficits and bothering symptoms that might otherwise pass unnoticed, lost in the bigger picture of a worrisome and potentially lethal pathology. All of the symptoms reported by our patients had already been described in the literature, and most of them fall within the expected percentages $[7,11,15,20]$. Notably we recorded a markedly lower percentage of patients reporting fever $(33 \%)$ in comparison to what is reported by others $(81,8-100 \%)$ [7]. This is not surprising, as most of our patients were diagnosed during the screening for Sars-Cov-2 infection among the personnel of our hospital, and none of them had been critically ill. Therefore, we consider our data concerning "typical" and general manifestation of the disease largely confirmative of what has already been reported by others. Also, critical illness in any case would markedly increase the chance of organ dysfunction from more general mechanisms, including hearing loss, so our study is not aimed to define audiovestibular sequelae of the whole Covid19 patient population, but only of non-hospitalized less critical patients.

Taste and smell related symptoms, in a cohort of patients including those enrolled in the present study have already been discussed elsewhere [18], with an upcoming article discussing follow up and persistence of those symptoms.

Hearing loss has been recently described as an atypical sign of Covid-19 [13]. In the present series, evaluating audiovestibular function in former Covid19 patients, a small percentage of subjects reported a worsening or a new onset of tinnitus (4.2\%) or subjective hearing loss $(8.3 \%)$, persistent in respec- tively $50 \%$ and $25 \%$ even after resolution of the main clinical picture. We performed a more in-depth analysis through a comparison of the patients' pure tone thresholds with an age-sex matched control group, in order to highlight any deviation from normality. While we found a significant difference for 3 of the investigated frequencies, namely $0.25,2$ and $4 \mathrm{kHz}$ (with 2 and $4 \mathrm{kHz}$ being slightly worse in the control group), the difference was minimal, and all patients fell within the range of normality for those frequencies. We did not find a significant difference for high frequencies as reported in the patients with active SARS-CoV-2 infection. None of the patients in the present series has an objectifiable hearing loss attributable to Covid-19. These results suggest that, if a cochlear damage is indeed present during COVID19 , it tends to be transitory.

Being hearing and balance two functions of the same audiovestibular system, we found it rational to evaluate both functions together, also to increase our ability to detect the purported Covid-19 mediated damage of inner ear hairy cells. Dizziness, as a generic symptom, has been reported by as much as $16.8 \%$ of patients in other series [11], it is present in $8.3 \%$ of patients in our series, while spinning vertigo occurred only in 1 patient during the late phase of the illness, and regressed by the time of the present data collection, without any residuate at vHIT. No clear reports of spinning vertigo in Covid-19 can be found in the literature. Also, this is, to our knowledge, the first reported instrumental evaluation of vestibular function in Covid-19 patients. A comparison of vHIT values of cases and controls gave back only one significant value, namely the vHIT gain of the right anterior canal. While such statistical difference is present, all the patients' values for that canal fell within the range of normality, and no covert or overt saccades were detected. Our data suggest that no clinically relevant signs of vestibular impairment can be found in former Covid-19 patients. Whether a transitory vestibular loss with subsequent full recovery is present in patients with COVID-19, and what is the exact cause of the reported vestibular symptoms, remains unknown, but mild vestibular symptoms such as dizziness and imbalance might simply be the result of the profound asthenia and fatigue often experienced by COVID-19 patients.

Our research has some shortcomings and sources of potential bias. It doesn't allow an easy and direct comparison with the only other paper covering audiological symptoms [13], mainly because we couldn't perform diagnostic TEOAEs at our facility, and we 
did not test patients during the active phase of the infection. However, while TEOAEs help define the site of the audiological lesion (cochlear vs retrocochlear), it doesn't add much to the definition of clinically relevant hearing loss in COVID-19 patients. Also, we only performed vHIT as a screening and diagnostic test for vestibular loss. While notoriously sensible only to damage affecting response to high frequency angular accelerations, vHIT has the advantage of minimal discomfort for the patients, quantifiable gain (numeric parameters), and examination of all semicircular canals and both branches of the vestibular nerve [13]. It is well known that vHIT is considered a complementary, and not alternative test with respect to other vestibular testing methods, and especially caloric balance, however caloric balance is more invasive, uncomfortable for the patient, and in the end less justifiable than vHIT in such a setting. For audiological testing we couldn't compare the test to baselines that were not available for the patients, therefore we used a control group to compare the results, a less effective, but also the only available method. We didn't calculate an appropriate sample size, opting instead to simply enroll all the patients that underwent our free screening that fitted the inclusion criteria for our observational study. Moreover, the adhesion was of course on voluntary basis, and did not represent a consecutive series of patients, but it did include most of the affected personnel of our Institution.

In conclusion, we think that even with all the above mentioned limitations, we can still state with a reasonable degree of confidence that even if an audiovestibular involvement was present during $\mathrm{CO}$ VID-19, no definite signs of such a damage can be seen in recently recovered COVID-19 patients, who had not been previously hospitalized.

\section{Acknowledgments}

Many thanks to Sara Palmas, Maria Lucia Piras, Maria Giovanna Canu and Maria Francesca Arca Sedda and Daniela Lucidi for their precious technical and scientific support.

\section{Conflict of interest}

The authors declare that they have no conflict of interest.

\section{References}

[1] L. Angelo Vaira, C. Hopkins, G. Salzano, M. Petroce1li, A. Melis, M. Cucurullo, M. Ferrari, L. Gagliardini, C. Pipolo, G. Deiana, V. Fiore, A. De Vito, N. Turra, S. Canu, A. Antonio Maglio, A. Serra, F. Bussu, G. Madeddu, S. Babudieri, A. Giuseppe Fois, P. Pirina, F.A. Salzano, P. De Riu, F. Biglioli and G. De Riu, Olfactory and gustatory function impairment in COVID-19 patients: Italian objective multicenter-study, Head Neck (2020). doi:10.1002/ hed.26269

[2] W.G. on M.P.-T.T. Audiometry, and Working Group on Manual Pure-Tone Threshold Audiometry, Guidelines for Manual Pure-Tone Threshold Audiometry, (2005). doi:10.1044/policy.g12005-00014

[3] J.F.-W. Chan, S. Yuan, K.-H. Kok, K.K.-W. To, H. Chu, J. Yang, F. Xing, J. Liu, C.C.-Y. Yip, R.W.-S. Poon, H.-W. Tsoi, S.K.-F. Lo, K.-H. Chan, V.K.-M. Poon, W.-M. Chan, J.D. Ip, J.-P. Cai, V.C.-C. Cheng, H. Chen, C.K.-M. Hui and K.-Y. Yuen, A familial cluster of pneumonia associated with the 2019 novel coronavirus indicating person-to-person transmission: a study of a family cluster, Lancet 395 (2020), 514-523.

[4] L.E. Gralinski and V.D. Menachery, Return of the Coronavirus: 2019-nCoV, Viruses 12 (2020). doi:10.3390/ v12020135

[5] C. Hopkins, P. Surda and N. Kumar, Presentation of new onset anosmia during the COVID-19 pandemic, Rhinology (2020). doi:10.4193/Rhin20.116

[6] J. Krajewska, W. Krajewski, K. Zub and T. Zatoński, COVID-19 in otolaryngologist practice: a review of current knowledge, European Archives of Oto-Rhino-Laryngology (2020). doi:10.1007/s00405-020-05968-y

[7] L.-Q. Li, T. Huang, Y.-Q. Wang, Z.-P. Wang, Y. Liang, T.-B. Huang, H.-Y. Zhang, W. Sun and Y. Wang, COVID19 patients' clinical characteristics, discharge rate, and fatality rate of meta-analysis, J Med Virol (2020). doi: $10.1002 /$ jmv. 25757

[8] S.-L. Liu, New virus in China requires international control effort, Nature 577 (2020) 472.

[9] L. Loffredo, F. Pacella, E. Pacella, G. Tiscione, A. Oliva and F. Violi, Conjunctivitis and COVID-19: a meta-analysis, $J$ Med Virol (2020). doi:10.1002/jmv.25938

[10] A. Lovato and C. de Filippis, Clinical Presentation of CO VID-19: A Systematic Review Focusing on Upper Airway Symptoms, Ear Nose Throat J (2020). 145561320920762.

[11] L. Mao, H. Jin, M. Wang, Y. Hu, S. Chen, Q. He, J. Chang, C. Hong, Y. Zhou, D. Wang, X. Miao, Y. Li and B. Hu, Neurologic Manifestations of Hospitalized Patients With Coronavirus Disease 2019 in Wuhan, China, JAMA Neurol (2020). doi:10.1001/jamaneurol.2020.1127

[12] L.A. McGarvie, H.G. MacDougall, G. Michael Halmagyi, A.M. Burgess, K.P. Weber and I.S. Curthoys, The Video Head Impulse Test (vHIT) of Semicircular Canal Function - Age-Dependent Normative Values of VOR Gain in Healthy Subjects, Frontiers in Neurology 6 (2015). doi:10.3389/fneur.2015.00154

[13] M.W.M. Mustafa, Audiological profile of asymptomatic Covid-19 PCR-positive cases, Am J Otolaryngol (2020), 102483.

[14] Y.R. Nobel, M. Phipps, J. Zucker, B. Lebwohl, T.C. Wang, M.E. Sobieszczyk and D.E. Freedberg, Gastrointestinal Symptoms and COVID-19: Case-Control Study from the United States, Gastroenterology (2020). doi:10.1053/j.gastro.2020.04.017 
[15] G. Pascarella, A. Strumia, C. Piliego, F. Bruno, R. Del Buono, F. Costa, S. Scarlata and F.E. Agrò, COVID-19 diagnosis and management: a comprehensive review, J Intern Med (2020). doi:10.1111/joim.13091

[16] A. Piras, D. Rizzo, S. Uzzau, G. De Riu, S. Rubino and F. Bussu, Inappropriate Nasopharyngeal Sampling for SARSCoV-2 Detection Is a Relevant Cause of False-Negative Reports, Otolaryngology-Head and Neck Surgery (2020) 019459982093179. doi:10.1177/0194599820931793

[17] M. Strupp, J.-S. Kim, T. Murofushi, D. Straumann, J.C. Jen, S.M. Rosengren, C.C. Della Santina and H. Kingma, Bilateral vestibulopathy: Diagnostic criteria Consensus document of the Classification Committee of the Bárány Society, Journal of Vestibular Research 27 (2017), 177-189. doi:10.3233/ves-170619

[18] L.A. Vaira, G. Deiana, A.G. Fois, P. Pirina, G. Madeddu, A. De Vito, S. Babudieri, M. Petrocelli, A. Serra, F. Bussu, E. Ligas, G. Salzano and G. De Riu, Objective evaluation of anosmia and ageusia in COVID-19 patients: Single-center experience on 72 cases, Head Neck (2020). doi:10.1002/hed.26204

[19] S.H. Wong, R.N. Lui and J.J. Sung, Covid-19 and the digestive system, J Gastroenterol Hepatol 35 (2020), 744-748.
[20] J.-J. Zhang, X. Dong, Y.-Y. Cao, Y.-D. Yuan, Y.-B. Yang, Y.-Q. Yan, C.A. Akdis and Y.-D. Gao, Clinical characteristics of 140 patients infected with SARS-CoV-2 in Wuhan, China, Allergy (2020). doi:10.1111/all.14238

[21] N. Zhu, D. Zhang, W. Wang, X. Li, B. Yang, J. Song, X. Zhao, B. Huang, W. Shi, R. Lu, P. Niu, F. Zhan, X. Ma, D. Wang, W. Xu, G. Wu, G.F. Gao, W. Tan and China Novel Coronavirus Investigating and Research Team, A Novel Coronavirus from Patients with Pneumonia in China, 2019, N Engl J Med 382 (2020), 727-733.

[22] WHO announces COVID-19 outbreak a pandemic, (2020). http://www.euro.who.int/en/health-topics/health-emergenc ies/coronavirus-covid-19/news/news/2020/3/who-announc es-covid-19-outbreak-a-pandemic (accessed May 19, 2020).

[23] Global research on coronavirus disease (COVID-19), (n.d.). https://www.who.int/emergencies/diseases/novelcoronavirus-2019/global-research-on-novel-coronavirus2019-ncov (accessed May 22, 2020).

[24] Degree of Hearing Loss, American Speech-LanguageHearing Association. (n.d.). https://www.asha.org/public/ hearing/Degree-of-Hearing-Loss/ (accessed May 25, 2020). 